\title{
La révolution américaine en territoire indien
}

The American Revolution in Indian Country

Colin G. Calloway

\section{(2) OpenEdition \\ 1 Journals}

Édition électronique

URL : https://journals.openedition.org/ahrf/11950

DOI : 10.4000/ahrf.11950

ISSN : 1952-403X

Éditeur :

Armand Colin, Société des études robespierristes

\section{Édition imprimée}

Date de publication : 1 mars 2011

Pagination : 131-150

ISBN : 978-2-200-92677-9

ISSN : 0003-4436

Référence électronique

Colin G. Calloway, "La révolution américaine en territoire indien », Annales historiques de la Révolution française [En ligne], 363 | janvier-mars 2011, mis en ligne le 01 mars 2014, consulté le 22 avril 2022. URL : http://journals.openedition.org/ahrf/11950; DOI : https://doi.org/10.4000/ahrf.11950 


\title{
LA RÉVOLUTION AMÉRICAINE EN TERRITOIRE INDIEN
}

Colin G. CALLOWAY

\begin{abstract}
Cet article met en perspective l'expérience des indigènes américains pendant la Révolution américaine, et leur lutte pour établir leur propre modèle d'indépendance, bien différent de celui des colons anglophones. L'auteur fixe particulièrement son regard sur les Iroquois de New York, les Shawnees et les Delawares de la vallée de l'Ohio, ainsi que sur les Cherokees du Sud. II insiste sur le regard que les colons ont porté sur la participation des Indiens à la Révolution américaine, montrant comment, l'indépendance proclamée, celle-ci a justifié leur exclusion de la nouvelle nation.
\end{abstract}

Mots-clés : Indiens américains, Cherokee, guerre de frontière, Iroquois, Révolution américaine, Shawnee.

La Déclaration d'Indépendance dépeint sans ambages le rôle des Indiens d'Amérique dans la Révolution américaine. Thomas Jefferson écrit que le roi George III, ajoutant à ses autres actes d'oppression, avait « cherché à attirer sur les habitants de nos frontières les Indiens, ces sauvages sans pitié, dont la manière bien connue de faire la guerre est de tout massacrer, sans distinction d'âge, de sexe ni de condition ». Inscrits dans le document fondateur des États-Unis, texte quasi sacré, ces mots de Jefferson plaçaient les Indiens du mauvais côté, et du combat pour la liberté, et de l'histoire, dès le début de la Révolution. Alors que les Américains se battaient pour leurs droits et leurs libertés, les indigènes d'Amérique, individus agressifs manœuvrés par un roi tyrannique, s'opposaient à eux. Cependant les affres de la mort d'un vieil ordre impérial, et les douleurs de l'enfantement d'une nation nouvelle en plein essor firent franchir de nouveaux paliers à 
la violence en territoire indien, où les attaques américaines à l'encontre des communautés indiennes furent également marquées par un massacre total « sans distinction d'âge, de sexe ni de condition ». Et les Indiens qui s'engagèrent dans le combat de la Révolution (certains en faveur des Américains mais la plupart du côté des Britanniques) ne se battaient pas contre la liberté : tout comme les patriotes américains, ils combattaient pour défendre leur liberté. Les offensives des Américains pour repousser leurs frontières constituaient une menace plus grande pour les terres, les libertés et les modes de vie des Indiens qu'un roi lointain. La guerre de l'Indépendance américaine fut aussi une guerre pour l'indépendance des Indiens.

La Révolution américaine exacerba, mais n'engendra pas, sur le front pionnier, une culture de violence raciale ni un héritage de haine vis-à-vis de l'Indien. Au cours de la première moitié du XVIII ${ }^{\mathrm{e}}$ siècle, en dépit de conflits périodiques, les Indiens et les colons étaient souvent parvenus à maintenir des relations fondées sur la coexistence et le compromis. Mais des colons, de plus en plus nombreux, empiétaient sur des terres indiennes; les tensions ethniques, sociales et politiques s'intensifièrent et la guerre de Sept Ans provoqua des effusions de sang, la terreur et la crainte ${ }^{1}$. À la fin de cette guerre, et une douzaine d'années avant que les colons américains ne se révoltent contre la GrandeBretagne, les Indiens de la vallée de l'Ohio et des Grands Lacs menèrent une guerre d'indépendance contre l'empire le plus puissant au monde. En 1763, récents conquérants de la Nouvelle France, les Britanniques se comportèrent comme des vainqueurs en terre indienne. Regimbant face à l'arrivée de garnisons britanniques et mécontents de n'avoir pas reçu de cadeaux de leur part - qui servaient à sceller des alliances et à assurer des relations de bonne foi - Pontiac des Ottawas, Guyashota des Senecas, Shingas des Delawares et d'autres chefs de guerre lancèrent un assaut commun à plusieurs tribus qui détruisit chacun des forts britan-

(1) James H. Merrell, Into the American Woods Negotiators on the Pennsylvania Frontier, New York, W.W. Norton, 1999; Jane T. MERritT, At the Crossroads Indians and Empires on a Mid-Atlantic Frontier, 1700-1763, Chapel Hill, University of North Carolina Press, 2003; Peter Silver, Our Savage Neighbors How Indian War Transformed Early America, New York, W.W. Norton, 2008; Kevin Kenny, Peaceable Kingdom Lost The Paxton Boys and the Destruction of William Penn's Holy Experiment, New York, Oxford University Press, 2009; Peter WAY, «The Cutting Edge of Culture : British Soldiers Encounter Native Americans in the French and Indian War », dans Martin Daunton et Rick HalPeRn (dir.), Empire and Others : British Encounters with Indigenous Peoples, 1600-1850, Philadelphia, University of Pennsylvania Press, 1999, p. 123-148; Matthew C. WARD, Breaking the Backcountry The Seven Years' War in Virginia and Pennsylvania, 1754-1765, Pittsburgh, University of Pittsburgh Press, 2003, p. 42-43, p. 55. 
niques à 1'ouest des Appalaches sauf Detroit, Niagara et Fort Pitt ${ }^{2}$. Le gouvernement des colonies à Londres réagit en déclarant que la chaîne des Appalaches constituait la frontière entre la colonisation britannique et les terres indiennes. La ligne définie par la proclamation royale ne fut jamais considérée comme permanente et c'est à peine si elle contint les empiétements sur les terres indiennes. L'armée ne pouvait pas la faire respecter, les colons n'en tenaient souvent pas compte et des spéculateurs fonciers comme Benjamin Franklin et George Washington, qui avaient espéré devenir riches en vendant des terres au-delà des Appalaches à des pionniers allant vers l'Ouest, faisaient pression pour qu'elle soit modifiée. Conçue pour mettre de l'ordre sur la frontière américaine, cette proclamation fut à l'origine d'une chaîne d'événements qui menèrent à la révolution et à l'indépendance, particulièrement après que la Grande-Bretagne eut décidé d'augmenter les impôts dans les colonies pour l'aider à administrer l'immensité de son nouvel empire en Amérique du Nord ${ }^{3}$.

Sir William Johnson, le surintendant britannique des affaires indiennes dans le Nord, négocia une nouvelle ligne frontière par le traité de Fort Stanwix en 1768. Les Iroquois réalisèrent une cession massive de terres, qui écartait la pression de leur propre territoire, par la vente de zones de chasse au sud de l'Ohio que réclamaient les Shawnees et les Cherokees. Avec de nouveaux traités sur la frontière établis avec les Cherokees au Sud, ceci ouvrit une vaste bande de territoire - la plus grande partie de la Virginie occidentale et du Kentucky actuels - qui, tel un poignard, pointait en direction du cœur des terres indiennes. Ainsi que s'en rendit compte le général Thomas Gage, le commandant en chef des forces britanniques en Amérique du Nord, la Grande-Bretagne pouvait établir des frontières où bon lui semblait mais « les habitants de la frontière sont trop nombreux, trop enclins à l'anarchie et à la licence pour être jamais maîtrisés ». La Grande-Bretagne ne pouvait les empêcher de pénétrer sur les terres indiennes et les chefs indiens ne pouvaient se faire

(2) Gregory Evans Down, War Under Heaven Pontiac, the Indian Nations and the British Empire, Baltimore, Johns Hopkins University Press, 2002; David Dixon, Never Come to Peace Again Pontiac's Uprising and the Fate of the British Empire in North America, Norman, University of Oklahoma Press, 2005; Richard Middleton, Pontiac's War : Its Causes and Consequences, New York, Routledge, 2007.

(3) Colin G. Calloway, The Scratch of a Pen 1763 and the Transformation of North America, New York, Oxford University Press, 2006; Woody Holton, Forced Founders Indians, Debtors, Slaves, and the Making of the American Revolution in Virginia, Chapel Hill, University of North Carolina Press, 1999, chap. 1. 


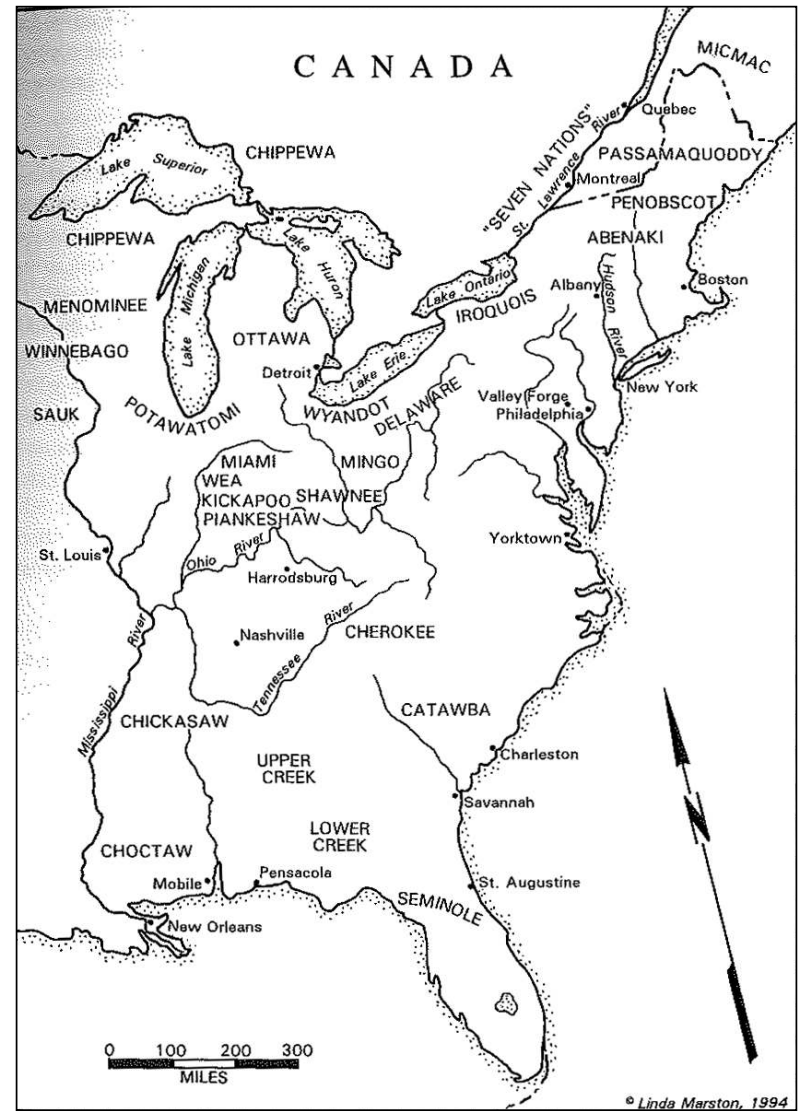

Les tribus indiennes pendant la Révolution américaine

obéir des jeunes guerriers que l'invasion de leurs territoires mettait en colère ${ }^{4}$. Lorsque le gouvernement donna l'ordre, en 1771, d'abandonner les forts de l'Ouest par mesure d'économie, ceci retira à l'armée britannique son «pouvoir de maîtrise » dans la région. En même temps, comme il ne parvenait pas à accorder un développement foncier limité au-delà de la ligne fixée par la proclamation et atermoyait sans cesse pour donner son approbation à différents plans afin d'accorder des propriétés, « le ministère cautionnait les aventuriers sans scrupules, impatients ou oppor-

(4) James Sullivan et alii (dir.), The Papers of Sir William Johnson, 14 vol., Albany, University of the State of New York, 1921-65, 6, p. 212; 12, p. 710. 
tunistes qui prendraient des initiatives pour la mise en valeur de l'Ouest et aggraveraient les désordres et conflits qui se manifestaient déjà à propos des terres de l'Ouest $»^{5}$.

Le traité de Fort Stanwix provoqua une invasion d'arpenteurs et de pionniers sur les territoires de chasse des Shawnees qui «mit en fureur tous leurs guerriers ». L'agent indien et négociant George Croghan évalua à quatre ou cinq mille le nombre des familles qui franchirent les montagnes en 1769 et tout au long du printemps et de l'été suivant des files de chariots parcoururent les routes en direction de l'Ohio ${ }^{6}$. Ainsi que l'expliqua Sir William Johnson au comte de Dartmouth : « Ces pionniers partent généralement avec un préjugé d'ensemble contre tous les Indiens, et les jeunes guerriers ou chasseurs indiens sont trop souvent disposés à user de représailles $»^{7}$. Il y avait, ainsi que Croghan l'exprima, « une disposition trop forte chez les occupants de la frontière à tuer les Indiens $\|^{8}$. Cette disposition se manifesta au printemps de 1774 lorsque des bandits de la frontière assassinèrent treize femmes et enfants membres de la famille de Tachnedorus, un chef mingo, également connu sous le nom de John Logan. Parmi les victimes se trouvaient l'épouse shawnee de Logan et sa sœur enceinte que les tueurs suspendirent par les poignets; ils lui ouvrirent le ventre et empalèrent sur un piquet l'enfant qu'elle portait. Le chagrin de Logan prit un caractère immortel dans différentes versions d'un discours qu'on lui attribua, particulièrement celle reprise par Thomas Jefferson dans son livre Notes sur l'État de Virginie. Lorsque Logan se vengea en organisant des raids contre des colons en Virginie, le gouverneur de cet État, John Murray, comte de Dunmore, et ses collègues saisirent l'occasion des incursions des Indiens pour battre le rappel et se mettre en guerre contre les Shawnees ${ }^{9}$. Moins nombreux et moins bien armés au cours de la bataille de Point Pleasant, les Shawnees furent contraints d'accepter une paix qui confirma la perte de leurs territoires de chasse au sud de 1'Ohio. Les chefs shawnees dirent aux Virginiens l'année suivante :

(5) Eric Hinderaker, Elusive Empires Constructing Colonialism in the Ohio Valley, 1763-1800, Cambridge, Cambridge University Press, 1997, p. 170.

(6) Albert T. Volwiler, George Croghan and the Westward Movement, 1741-1782, Cleveland, Arthur H. Clark Co., 1926, p. 223-224; Sullivan et alii (dir.), The Papers of Sir William Johnson, op. cit., 7, p. 185.

(7) Edmund B. O'Callaghan (dir.)., Documents Relating to the Colonial History of the State of New York, 15 vol., Albany, Weed, Parsons, and Co., 1853-57, 8, p. 396.

(8) «Letters of Colonel George Croghan», Pennsylvania Magazine of History and Biography, 15, 1891, p. 437-438.

(9) Reuben Gold Thwaites et Louise Phelps Kellogg (dir.), Documentary History of Dunmore's War, 1774, Madison, Wisconsin Historical Society, 1905. 
« Nous sommes souvent enclins à croire qu'il n'y a aucun lieu pour notre repos et que vos intentions étaient de nous dépouiller complètement de notre pays tout entier $»^{10}$.

Alors que les combats sur la frontière des terres cédées à Fort Stanwix se confondirent avec le conflit de la Révolution américaine, la vallée de l'Ohio devint une zone vivement contestée, puis un champ de la mort. Henry Hamilton, le commandant britannique de Detroit, et George Morgan, l'agent américain à Fort Pitt, rivalisèrent pour obtenir la soumission des tribus. Les agents britanniques et américains promirent protection ou extermination aux Indiens susceptibles de se trouver sous leurs feux croisés et les Indiens savaient que leurs terres et leurs vies étaient en jeu. Contrairement à ce que Jefferson affirmait dans la Déclaration d'Indépendance, la plupart ne s'emparèrent cependant pas immédiatement de leurs tomahawks et n'entrèrent pas en guerre pour le roi. Ils essayèrent de rester neutres dans un conflit qu'ils considérèrent, au début, comme une bataille entre Anglais. Les anciens chefs civils ou de villages qui conseillaient la neutralité et la modération étaient souvent contestés ou ignorés par des chefs de guerre plus jeunes qui appelaient à l'action militante. La Révolution américaine divisa les Indiens comme elle partagea les colons et ces divisions donnèrent parfois lieu à des conflits d'Indiens contre Indiens ${ }^{11}$.

Samson Occom, le pasteur indien mohegan qui, dix ans auparavant, avait effectué une tournée en Grande-Bretagne, collectant des fonds pour aider à la création de Dartmouth College, regrettait que les Blancs ne laissent pas les Indiens en dehors de la guerre : «Qu'en ont-ils à faire de vos querelles »? demandait-il ${ }^{12}$. Mais la neutralité n'était pas une option viable pour les Indiens. Le chef shawnee Cornstalk avait mené ses guerriers dans la guerre de Dunmore mais conseillait dorénavant une position neutre et cultivait des relations pacifiques avec les Américains. Capturé sous un drapeau de trêve à Fort Randolph, il fut assassiné par une milice américaine en 1777, tout comme son fils et un autre Shawnee dont le corps avait été «terriblement mutilé ». La sœur de Cornstalk, Nonhelema, poursuivit son travail pour la paix et aida les Américains

(10) Robert L. SCRIBNER et alii (dir), Revolutionary Virginia, The Road to Independence A Documentary Record, 7 vol., University Press of Virginia, 1973-83, 7, p. 770.

(11) Colin G. Calloway, The American Revolution in Indian Country Crisis and Diversity in Native American Communities, Cambridge, Cambridge University Press, 1995.

(12) Harold Blodgett, Samson Occom, Hanover, N.H., Dartmouth College Publications, 1935 , p. 164-165. 
mais la plupart des Shawnees faisaient désormais cause commune avec les Britanniques qui les avaient prévenus que les Américains avaient l'intention de les anéantir. L'assassinat de Cornstalk fit grossir les rangs des militants shawnees. Les courriers shawnees portaient des ceintures de guerre et la nouvelle de la mort de Cornstalk à travers le territoire indien attira des alliés aussi éloignés que les Ojibwas et les Creeks pour donner la plus étendue des coalitions entre tribus indiennes jusque-là formées ${ }^{13}$. Des guerriers shawnees attaquèrent des établissements américains dans le Kentucky et dans la vallée de l'Ohio et fermèrent effectivement la rivière Ohio à la circulation des Américains. Les assauts conjugués des guerriers indiens et des patrouilleurs britanniques causèrent l'épouvante et la ruine dans les établissements américains sur la frontière et les Shawnees et leurs alliés "réglèrent d'anciens comptes avec une effroyable efficacité $\gg{ }^{14}$.

Mais les Indiens n'avaient pas le monopole de l'atrocité et de la terreur. Les colonies proposaient régulièrement des primes pour des scalps d'Indiens; Henry Hamilton, le lieutenant-gouverneur britannique à Détroit, était tristement célèbre en tant que " général acheteur de cheveux », et George Rogers Clark, celui qui causerait sa chute, affirmait que « la seule façon de faire la guerre aux Indiens » était « de les surpasser en cruauté »; c'est cette philosophie qu'il mit en pratique avec force détails saisissants au siège de Vincennes en 1779 lorsqu'il donna l'ordre que les Indiens capturés soient attachés et tués à coups de haches et que leurs corps soient jetés à la rivière, puis ordonna à ses hommes de scalper un prisonnier canadien, le tout sous les yeux des soldats de la garnison britannique ${ }^{15}$. En 1780, Clark envahit le territoire shawnee avec un millier d'hommes. Les Shawnees brûlèrent leur ville de Chillicothe plutôt que de la laisser tomber aux mains des " Grands Couteaux » mais à Piqua, sur la rivière Mad, ils restèrent sur leurs positions et ne se retirèrent pas avant que Clark n'ait pointé son canon de six livres vers la maison des conseils du village dans laquelle de nombreux habitants avaient trouvé refuge. Les pertes des Shawnees furent faibles mais les Américains tuèrent quelques vieillards qu'ils trouvèrent cachés dans les champs de maïs et ils passèrent

(13) Gregory Evans Dowd, A Spirited Resistance The North American Indian Struggle for Unity, 1745-1815, Baltimore, Johns Hopkins University Press, 1992, chap. 3.

(14) Patrick GRIFFIn, American Leviathan Empire, Nation, and Revolutionary Frontier, New York, Hill and Wang, 2007, chap. 6, cité p. 159.

(15) Milo M. QuaIFE (dir.), The Conquest of Illinois, Chicago, R.R. Donnelley and Sons, 1920, p. 148-149, p. 167-169; James Alton James (dir.), George Rogers Clark Papers, 1771-1781, Springfield, Illinois State Historical Society, 1912, p. 144, 167, 189. 
trois jours à brûler les récoltes. Quelques hommes pillèrent des tombes shawnees pour s'emparer d'objets funéraires et de scalps ${ }^{16}$. La milice du Kentucky traversa la rivière Ohio presque chaque année pour mettre le feu aux villages et récoltes des Shawnees. Thomas Jefferson voulait que ces derniers soient exterminés ou expulsés de leurs terres et il préconisait de retourner d'autres tribus contre eux ${ }^{17}$.

En 1782, des guerriers shawnees et d'autres tribus mirent en déroute Daniel Boone et une armée d'hommes du Kentucky à la bataille de Blue Licks, au cours de laquelle un des fils de Daniel Boone trouva la mort. À l'automne, Boone accompagna George Rogers Clark pour une nouvelle invasion de représailles en territoire shawnee. Boone raconta que l'expédition incendia cinq villages, " détruisit entièrement leur maïs et autres récoltes, et répandit la désolation à travers tout leur pays ». La plupart des guerriers étaient absents lorsque Clark lança son attaque et les hommes âgés, les femmes et les jeunes résistants refusèrent d'être mêlés à une bataille ouverte. À l'évidence ils essuyèrent peu de pertes mais, d'après certains récits, les hommes du Kentucky, que les Indiens appelaient « les sauvages blancs de Virginie », commirent des atrocités ${ }^{18}$.

Confrontés à la perspective d'une guerre continue et d'une invasion quasi annuelle dans l'Ohio, de nombreux Shawnees partirent en direction de l'Ouest vers ce qui est à présent le Missouri, et que l'Espagne revendiquait. D'autres se rendirent au Sud, dans les territoires cherokee et creek. Ceux qui restèrent se replièrent pour éviter les attaques américaines et se rassemblèrent dans des nouvelles villes sur les rivières Auglaize et Maumee dans le nord-est de l'Ohio. Dès la fin de la Révolution, les Shawnees et leurs alliés étaient concentrés dans le coin nord-ouest de l'Ohio, dans des communautés qui avaient été créées par la guerre et qui vivaient sur le pied de guerre ${ }^{19}$.

Comme les Shawnees leurs voisins dans la vallée de l'Ohio, les Delawares furent au début peu disposés à prendre les armes ou à soutenir les Britanniques. Ils tentèrent de conserver leur rôle et leur réputation en tant qu'artisans de la paix et bâtisseurs d'alliances et refusèrent

(16) Des récits de l'expédition sont rassemblés dans Clark's Shawnee Campaign of 1780 Contemporary Accounts, J. Martin West (dir.), Springfield, OH, the Clark County Historical Society, 1975.

(17) Julian P. Boyd (dir.), The Papers of Thomas Jefferson, 35 vol. à ce jour, Princeton University Press, 1950, vol. 3, p. 259, 276.

(18) «Journal of Daniel Boone », Ohio Archaeological and Historical Publications 13, 1904, p. 276; Draper Mss, State Historical Society of Wisconsin, (microfilm), 1AA, p. 276-277.

(19) Colin G. Calloway, The American Revolution in Indian Country...op. cit., chap. 6. 
de donner une aide militaire directe à aucun des camps. White Eyes, chef des Delawares, conduisit son peuple pour conclure le traité de Fort Pitt en 1778, qui fut le premier signé par la nouvelle nation, et dans lequel les Delawares et le Congrès des États-Unis s'accordaient pour une alliance défensive. Mais d'autres Delawares se plaignirent que le traité était " écrit de manière perfide » : les Américains avaient incité leurs chefs à « signer certains écrits [...] lesquels, ils l'ont constaté depuis, leur avaient été interprétés de manière erronée et comportaient des déclarations et des engagements auxquels ils n'avaient jamais eu l'intention de souscrire ou de participer ». Puis des miliciens américains assassinèrent White Eyes, leur meilleur ami dans le territoire indien de l'Ohio. Les autorités américaines annoncèrent qu'il était mort de la variole mais le mal était fait. Comme les Shawnees après l'assassinat de Cornstalk, les Delawares déterrèrent la hache de guerre et le conflit avec la GrandeBretagne devint le leur ${ }^{20}$.

Les Américains ripostèrent - à l'aveuglette. En 1782, une troupe de miliciens américains entra dans la ville de Gnadenhütten, où la communauté composée surtout d'Indiens delawares avait été convertie par l'Église morave. Ils étaient chrétiens et pacifistes. Mais tout ce qui importait à la milice était le fait qu'ils étaient Indiens. Les Américains les répartirent en trois groupes : hommes, femmes et enfants. Puis, les Indiens s'étant agenouillés et chantant des hymnes, ils s'emparèrent de maillets de boucher et matraquèrent à mort quatre-vingt seize personnes. Gnadenhütten signifie « Huttes de Grâce ». Les guerriers delawares, qui combattaient dorénavant comme alliés des Britanniques, se vengèrent du massacre de leurs familles lorsque des soldats américains tombèrent entre leurs mains. Les récits de la torture rituelle et de l'exécution horrible du colonel William Crawford, ami de George Washington, et, comme lui, spéculateur foncier, donnèrent lieu à une propagande anti-indienne de grande portée, et alimentèrent le sentiment anti-indien. Dans le désordre et le chaos de la Révolution sur la frontière de la vallée de l'Ohio, les meurtres par représailles se multiplièrent et, selon les mots de l'historien Patrick Griffin, la violence d'origine raciste devint « la nouvelle base de la société dans l'Ouest $»^{21}$.

(20) Amy C. Schutt, Peoples of the River Valleys The Odyssey of the Delaware Indians, Philadelphia, University of Pennsylvania Press, 2007, p. 163-168; Colin G. Calloway (dir.), The World Turned Upside Down Indian Voices from Early America, Boston, Bedford Books, 1994, p. 156.

(21) Patrick Griffin, American Leviathan..., op. cit., p. 154. 
Dans le Sud, la Révolution divisa les Cherokees d'après des critères de générations. Pendant plus d'un demi siècle, les Cherokees avaient vu l'étendue de leurs terres dans le nord de la Georgie, l'est du Tennessee, l'ouest des Carolines du Nord et du Sud s'amenuiser traité après traité avec les colonies, et le rythme de leurs pertes territoriales s'accéléra de façon alarmante à la fin des années 1760 et dans les années 1770. En donnant leur accord pour vendre des terres, il se peut que les chefs les plus anciens aient tenté d'asseoir leur sécurité au moyen d'une zone tampon de terres cédées, tout en entretenant, dans le même temps, le commerce et les obligations réciproques avec les colons. Mais la politique d'apaisement des chefs courrouçait leurs fils et neveux. Sacrifier fréquemment des territoires de chasse sans combat privait les jeunes hommes de la possibilité de démontrer leurs talents de guerriers et de chasseurs, ce qui, dans la société cherokee, donnait aux hommes leur prestige, et ils le reprochaient aux chefs.

En mars 1775, au traité de Sycamore Shoals dans le Tennessee, le juge Richard Henderson et un groupe de spéculateurs fonciers de Caroline du Nord connu sous le nom de Transylvania Land Company persuada Attakullakulla, le chef principal Oconostota, et le "Raven de Chota », de vendre 27000 miles carrés de terres entre les rivières Kentucky et Cumberland - la plus grande partie du Kentucky moderne - en échange d'une cabane pleine de marchandises. Attakullakulla, le «deuxième homme » de la nation Cherokee, était connu des Blancs sous le nom de « Petit Charpentier» du fait de ses aptitudes de diplomate à élaborer des accords. On raconte que, dégoûté, un chef de guerre nommé Tsi'yugûnsini ou Dragging Canoe quitta en trombe l'assemblée du traité, jurant qu'il rendrait « sombres et ensanglantées » les terres cédées. Il dit aux Britanniques «qu'il n'était pas intervenu dans ces marchandages mais il en rendait responsables certains de leurs anciens qui, d'après lui, étaient trop âgés pour chasser, et qui, à cause de leur pauvreté, avaient été persuadés de vendre leur terre, mais que, pour sa part, il avait un grand nombre de jeunes hommes pour le soutenir, et qu'ils étaient déterminés à avoir leur terre $»^{22}$. Dragging Canoe était le fils d'Attakullakulla (ou, selon certains spécialistes, son neveu; dans la société matrilinéaire des Cherokees, le frère de la mère était habituellement le personnage masculin le plus important dans la vie d'un garçon).

(22) The Colonial and State Records of North Carolina, 30 vol., Raleigh, P. H. Hale, 1886-1907, vol. 10, p. 764. 
Le déclenchement de la Révolution procura aux guerriers cherokees une occasion de chasser les intrus hors de leur terre et de l'emporter dans les conseils de la nation Cherokee où les anciens maintenaient leur emprise. En mai 1776, quatorze délégués des nations du Nord (Shawnees, Delawares, Mohawks, Nanticokes et Ottawas) arrivèrent dans la « ville bien aimée » cherokee de Chota sur le Petit Tennessee. Réunis avec les chefs et guerriers cherokees dans la maison des assemblées, ils les exhortèrent à prendre les armes pour mener, ensemble, une guerre de résistance contre les Américains. Le délégué shawnee reprit les doléances de son peuple aux mains des Virginiens et rappela à ses auditeurs la manière dont le grand peuple des Indiens avait été réduit à une poignée en seulement quelques années. "Il est préférable de mourir comme des hommes que de décliner jusqu'à disparaître pouce après pouce », déclara-t-il. Il offrit une ceinture wampum de neuf pieds de long, peinte en rouge, en signe de guerre. Dragging Canoe s'avança et se saisit de la ceinture. Il frappa de sa hache le poteau de la guerre et entonna le chant de guerre. D'autres guerriers firent de même. Ce faisant, ils acceptaient symboliquement l'invitation à faire la guerre et déclaraient leur indépendance vis-à-vis des anciens chefs qui, normalement, auraient guidé les Cherokees dans des décisions aussi cruciales. Attakullakulla, Oconostota et leur génération se souvenaient de la manière dont les Britanniques avaient brûlé leurs récoltes et leurs villages la dernière fois que les Cherokees s'étaient mis en guerre et ils étaient maintenant opposés à combattre les Américains. Mais des années de ventes de terres avaient émoussé leur autorité et, « au lieu de s'opposer à l'irréflexion des jeunes gens avec fougue, [ils] s'assirent, abattus et silencieux $»^{23}$.

La Révolution marqua aussi une nette divergence entre les Cherokees et leurs voisins colons car les guerriers dans les deux sociétés «se rendaient maîtres du sentier $»^{24}$. La guerre fut un désastre pour les Cherokees. Les troupes de milices de Virginie, des Carolines du Nord et du Sud, et de Georgie avancèrent rapidement en territoire cherokee. « Faites un travail propre sur votre route », comme en donna l'ordre aux troupes William Henry Drayton, juge suprême de la Caroline du Sud; « coupez tous les champs de maïs indiens et brûlez chaque ville indienne ».

(23) K.G. Davies (dir.), Documents of the American Revolution, 1770-1783, 21 vol., Shannon, Irish University Press, 1972-1982, vol. 12, p. 203-204; Colonial and State Records of North Carolina, vol. 10, p. 777-780.

(24) Tom Hatley, The Dividing Paths Cherokees and South Carolinians through the Era of Revolution, New York, Oxford University Press, 1993, p. 228, 231. 
Chaque Indien capturé deviendrait « l'esclave et la propriété de celui qui l'avait pris ». Le but de la campagne était clair et simple : « Que la nation soit éradiquée, et que les terres deviennent propriété publique ». Thomas Jefferson espérait que les Cherokees seraient «maintenant chassés audelà du Mississippi ». Les soldats américains détruisirent des récoltes, incendièrent des villes et repoussèrent leurs habitants vers les bois ${ }^{25}$.

Confrontés à une destruction massive, les anciens chefs réaffirmèrent leur autorité et sollicitèrent la paix. Ils reprochèrent à Dragging Canoe et aux jeunes guerriers dont ils n'avaient pu se faire obéir d'avoir déclenché la guerre ${ }^{26}$. Au printemps 1777, Attakullakulla, Oconostota et une délégation de trente Cherokees se rendirent à Williamsburg pour des pourparlers de paix. Les Cherokees des régions basses conclurent la paix avec la Caroline du Sud et la Georgie. Au traité de DeWitt's Corner avec la Georgie et la Caroline du Sud en mai et au traité de Long Island sur l'Holston avec la Virginie et la Caroline du Nord en juillet, les Cherokees se dessaisirent, pour prix de la paix, de plus que cinq millions d'arpents. Dragging Canoe ne participa pas aux négociations du traité27.

Avec ses partisans, il descendit vers le Sud et l'Ouest, sur la rivière Tennessee, construisit de nouvelles villes sur la rivière Chickamauga Creek et poursuivit le combat. En 1779, le colonel Evan Shelby mena un grand nombre de Virginiens le long du Tennessee et attaqua les villes sur la Chickamauga alors que Dragging Canoe et la plupart des guerriers étaient à l'assaut d'établissements sur la frontière en Georgie et en Caroline du Sud. Femmes et enfants s'échappèrent dans les bois mais Shelby brûla onze villes et détruisit quelques 20000 boisseaux de maïs. Dragging Canoe et ses partisans descendirent davantage le cours de la rivière et reconstruisirent leurs villes qui continuèrent à attirer « les jeunes pleins d'énergie, en provenance de presque chacune des villes de la nation $»^{28}$. Nées dans les combats, comme les communautés indiennes regroupées dans le nord-ouest de l'Ohio, les villes sur la Chickamauga vivaient sur le pied de guerre.

Les guerriers chickamaugas faisaient des incursions sur le front pionnier américain et se retiraient dans leurs forts; les Américains se ven-

(25) Drayton cité par Tom Hatley, The Dividing Paths..., op. cit., p. 192; Julian Boyd (dir.), The Papers of Thomas Jefferson..., op. cit., vol. 1, p. 494.

(26) Cynthia Cumfer, Separate Peoples, One Land The Minds of Cherokees, Blacks, and Whites on the Tennessee Frontier, Chapel Hill, University of North Carolina Press, 2007, p. 103.

(27) Draper Mss, 4QQ, 151-154.

(28) Walter Lowrie et Matthew St. Clair Clarke (dir.), American State Papers, Class II Indian Affairs, 2 vol., Washington, D.C., Gales and Seaton, 1832, vol. 1, p. 432. 
geaient par des représailles contre les villes plus accessibles des Cherokees qui tentaient de vivre en paix : elles présentaient des cibles plus faciles que celles des combattants de la liberté chickamaugas. Jefferson, désormais gouverneur de la Virginie, envoya des troupes à la fin de 1780 dans le territoire cherokee sous les ordres d'Arthur Campbell et John Sevier. Le chef de la ville de Chota dit que les Virginiens « teignirent leurs mains du sang d'un grand nombre de nos femmes et enfants, brûlèrent dix-sept villes, détruisirent toutes nos provisions, ce qui fit que nos familles et nousmêmes avons presque péri de famine $»^{29}$. Au printemps suivant, Sevier incendia encore quinze villes cherokees. Un nombre plus important de guerriers se joignit aux rangs des Chickamaugas du fait de ces attaques. Environ cent Shawnees s'y ajoutèrent aussi et certains Chickamaugas partirent vers le Nord pour vivre et se battre avec les Shawnees. La lutte des Chickamaugas se fondit dans une guerre indienne plus vaste pour l'indépendance dans l'Ouest qui se poursuivit pendant une douzaine d'années après que les Américains eurent gagné le combat pour leur indépendance à l'Est.

La tension raciale avait entraîné une effusion de sang et des meurtres par représailles sur les fronts pionniers de Pennsylvanie et de Virginie au milieu du siècle mais, sur les limites du territoire iroquois, des modèles de coexistence et d'acceptation culturelle entre les colons et les communautés indiennes s'étaient maintenus ${ }^{30}$. Les six nations de la Ligue iroquoise au nord de l'État de New York - Mohawk, Oneida, Onondaga, Cayuga, Seneca et Tuscarora - composaient la puissance amérindienne dominante dans le nord-est de l'Amérique du Nord. Au cours d'une grande partie du XVIII ${ }^{e}$ siècle, elles avaient usé de leur influence et montré leur force en diplomatie vis-à-vis des colonies et des autres tribus, jouant les puissances coloniales rivales les unes contre les autres ${ }^{31}$. Mais la Révolution mit en pièces l'unité de la Ligue des Iroquois et il en résulta une guérilla acharnée et destructrice entre les anciens voisins. Les Mohawks, avec le chef de guerre Joseph Brant en tête, soutenaient la couronne, du fait, en grande partie, de l'influence de Sir William Johnson, qui avait vécu parmi les Mohawks pendant des années et joua un rôle clef dans les relations entre

(29) Cité par James H. O’Donnell, The Southern Indians in the American Revolution, Knoxville, University of Tennessee Press, 1973, p. 118-119.

(30) David L. Preston, The Texture of Contact European and Indian Settler Communities on the Frontiers of Iroquoia, 1667-1783, Lincoln, University of Nebraska Press, 2009.

(31) Timothy J. Shannon, Iroquois Diplomacy on the Early American Frontier, New York, Penguin, 2008. 
Britanniques et Iroquois jusqu'à sa mort en 1774. Les Oneidas, voisins des Mohawks, penchaient en faveur des colonisateurs. Samuel Kirkland, leur missionnaire presbytérien/congrégationaliste était partisan d'une rupture avec l'Église anglicane, mais, en tant que nation iroquoise la plus directement menacée par l'expansion américaine, les Oneidas choisirent une stratégie consistant à s'aligner sur leurs voisins américains plutôt que de s'attirer leur hostilité. À la bataille sanglante de Oriskany en 1777, les Oneidas combattirent aux côtés des Américains, alors que Mohawks et Senecas étaient avec les Britanniques, ce qui constitua un événement destructeur pour la société iroquoise fondée sur des liens de clans et de parenté ${ }^{32}$.

Comme les Shawnees et les Cherokees, de nombreux Iroquois perdirent leurs foyers pendant la Révolution. Les Mohawks furent chassés de la vallée de la Mohawk et les Oneidas qui fuyaient devant les représailles occupèrent des camps de réfugiés misérables autour de Schenectady (New York). En 1779, George Washington dépêcha le général John Sullivan pour mener une campagne de terre brûlée en territoire iroquois. Les troupes de Sullivan incendièrent quarante villes iroquoises, abattirent des vergers et détruisirent environ 160000 boisseaux de maiis ${ }^{33}$. Sans abri ni nourriture à leur disposition, des milliers d'Iroquois s'enfuirent pour gagner le fort britannique à Niagara. Mais Niagara se trouvait à l'extrémité d'une longue voie de ravitaillement qui était fermée pendant les mois d'hiver lorsque les bateaux en provenance de Montréal et de Québec ne pouvaient naviguer sur les Grands Lacs pris par les glaces. Les réfugiés à Niagara souffrirent du froid, de famine, de maladie et de détresse au long de l'un des hivers les plus rigoureux jamais enregistré. Les guerriers iroquois reprirent leurs attaques contre les établissements américains sur les frontières de New York et de Pennsylvanie afin de s'emparer de céréales et d'animaux tout autant que de scalps et de prisonniers ${ }^{34}$.

(32) Alan TAYLOR, The Divided Ground Indians, Settlers, and the Northern Borderland of the American Revolution, New York, Alfred Knopf, 2006; Barbara GraYMONT, The Iroquois in the American Revolution, Syracuse, Syracuse University Press, 1872; Joseph T. GlatthaAr et James Kirby Martin, Forgotten Allies The Oneida Indians and the American Revolution, New York, Hill and Wang, 2006.

(33) Frederick CooK (dir), Journals of the Military Expedition of Major General John Sullivan against the Six Nations, Auburn, N.Y., Knapp, Peck, and Thompson, 1887; Max M. MinTz, Seeds of Empire The American Revolutionary Conquest of the Iroquois, New York, New York University Press, 1999; Joseph R. Fischer, A Well-Executed Failure The Sullivan Campaign against the Iroquois, July-September 1779, Columbia, South Carolina University Press, 1997.

(34) Colin G. Calloway, American Revolution in Indian Country..., op. cit., chap. 5. 
À la fin de la guerre, de nombreux Iroquois se réimplantèrent au nord de la nouvelle frontière, au Canada, plutôt que de rester dans l'État de New York et d'avoir à faire avec les Américains. Joseph Brant et ses partisans occupèrent des terres que le gouvernement britannique leur avait accordées sur la Grand River en Ontario, ce qui fut à l'origine de la Réserve des Six-Nations. Les Iroquois qui restèrent sur le territoire alors revendiqué par les États-Unis eurent à négocier avec le gouvernement fédéral, avec celui de l'État et avec des spéculateurs fonciers. Les commissaires américains au traité soutenus par des troupes américaines imposèrent leurs termes aux délégués iroquois au second traité de Fort Stanwix en 1784. Les Senecas restèrent sur leur territoire ancestral, à Tonawanda et Buffalo Creek, par exemple, mais alors qu'ils étaient les anciens maîtres de la région, ils devaient dorénavant se battre pour survivre dans un monde nouveau dominé par les Américains. Les Oneidas, qui avaient reçu des promesses de leurs alliés américains que leurs terres seraient protégées après la victoire de la révolution, perdirent des milliers d'arpents par des traités avec l'État de New York et avec des compagnies foncières. Au cours des années entre la Révolution et la Guerre de Sécession, les politiciens de l'État de New York, les intérêts liés au transport et les spéculateurs fonciers conspirèrent pour transformer la patrie des Iroquois en biens immobiliers américains. Les canaux, les chemins de fer, l'afflux massif de pionniers et le développement rapide de villes comme Buffalo métamorphosèrent ce qui avait, dans le passé, été l'Iroquoia ${ }^{35}$.

La violence de la Révolution ne cessa pas dans l'Ouest, bien après qu'elle se fut calmée dans l'Est. Les combats entre les rebelles et les soldats britanniques s'arrêtèrent effectivement après que Lord Cornwallis se fut rendu à l'armée de Washington et ses alliés français à Yorktown en 1781 mais les Indiens poursuivirent la guerre pour leur indépendance. Au traité de paix de Paris en avril 1783, la Grande-Bretagne reconnut l'indépendance des États-Unis et abandonna ses revendications sur tout le territoire compris entre l'Atlantique et le Mississippi et entre les Grands Lacs et la Floride mais la paix n'apporta pas la pacification en territoire indien. Les Indiens étaient furieux et incrédules lorsqu'ils apprirent que leurs alliés britanniques avaient donné leurs territoires. Les Américains leur dirent: " Vos pères les Anglais ont fait la paix avec nous pour euxmêmes, mais ils vous ont oubliés, vous leurs enfants qui avez combattu

(35) Laurence M. Hauptman, Conspiracy of Interests. Iroquois Dispossession and the Rise of New York State, Syracuse, Syracuse University Press, 1999. 
avec eux, et négligés comme des bâtards $»^{36}$. Il était difficile aux Indiens de ne pas être d'accord.

Cependant, comme ils s'attendaient bien à une nouvelle guerre avec la jeune république, les Britanniques au Canada maintinrent des alliances avec les Indiens pendant des années après la Révolution et continuèrent à les prévenir que les Américains avaient l'intention de leur ôter et leurs territoires, et la vie ${ }^{37}$. Il était difficile aux Indiens de ne pas être d'accord là-dessus aussi. Les efforts faits pour restaurer la paix et l'ordre social sur la frontière à la suite de la sanglante guerre révolutionnaire s'avérèrent futiles. Le nouveau gouvernement fédéraliste se trouvait face aux mêmes difficultés qui avaient beaucoup nui à l'administration impériale britannique dans ses tentatives pour imposer quelque retenue à ses citoyens alors qu'ils déferlaient sur les terres indiennes. Il trouva, à l'instar des Britanniques en 1763, que proclamer l'ordre sur une frontière éloignée n'avait rien à voir avec la possibilité de le maintenir. Une délégation de deux cents Indiens qui se rendirent à Saint-Louis au cours de l'été 1784 informa le gouverneur espagnol que les Américains s'abattaient sur la vallée de l'Ohio "comme une invasion de sauterelles", en chassaient les Indiens et construisaient des villes à des endroits où, dans le passé, se trouvaient des villages indiens. La Révolution, victorieuse pour les Américains, signifiait, d'après eux, la prolongation de la guerre et de la famine pour les Indiens; c'était « le plus grand coup qui avait pu [leur] être porté $»^{38}$.

L'avenir, voire peut-être la survie de la nouvelle nation, reposait sur son expansion et l'acquisition de terres indiennes. Le gouvernement des États-Unis n'avait pas de fonds après cette longue guerre; sa seule ressource était les territoires que la Grande-Bretagne avait cédés à la paix de Paris - des terres indiennes qui, transformées en «terres publiques », pouvaient être vendues à des colons pour aider à remplir le Trésor. À partir de la Déclaration d'Indépendance, les États-Unis tentèrent de définir leur position en tant que nouvelle nation au moyen de tractations diplomatiques avec les nations existant dans le monde. Cela impliquait de traiter avec les

(36) Correspondance et Papiers du gouverneur general, Sir Frederick Haldimand, 1758-91, British Museum, Additional Manuscripts, 21779, p. 117.

(37) Colin G. Calloway, Crown and Calumet: British-Indian Relations, 1783-1815, Norman, University of Oklahoma Press, 1987; Timothy T. WILLIG, Restoring the Chain of Friendship British Policy and the Indian of the Great Lakes, 1783-1815, Lincoln, University of Nebraska Press, 2008. VI, p. 281.

(38) Cité par Colin G. Calloway, The American Revolution in Indian Country..., op. cit., 
nations indiennes à l'Ouest ainsi qu'avec les nations européennes à l'Est ${ }^{39}$. Mais les négociations avec les nations indiennes concernaient les terres indiennes. À Fort Stanwix avec les Iroquois en 1784, à Fort McIntosh avec les tribus de l'Ohio en 1785 et à Fort Finney avec les Shawnees en 1786, les commissaires américains dictèrent des termes fondés sur la supposition que les États-Unis avaient déjà acquis le territoire indien, de droit, l'ayant conquis sur la Grande-Bretagne. À Fort McIntosh, ils informèrent les Indiens que, puisque le roi anglais n'avait rien prévu pour eux, il leur revenait « d'obtenir la paix des États-Unis [...] d'après les conditions qui semblaient convenables au Congrès ». Les objections des Indiens selon lesquelles ces terres leur avaient été transmises par leurs ancêtres étaient sans importance, dirent-ils, " parce que nous revendiquons le pays au titre de la conquête, et sommes à même de donner, pas de recevoir ». Au traité de Fort Finney en 1786, les commissaires américains, Richard Butler et George Rogers Clark, passèrent outre aux anciens rituels de la diplomatie de la ceinture wampum et proposèrent aux Shawnees un choix sans nuances entre l'acquiescement et la destruction ${ }^{40}$.

Il n'y eut pas de paix, même pour ceux qui signèrent les accords. Dans les mois qui suivirent le traité de Fort Finney, les miliciens du Kentucky étaient de retour en pays shawnee, effectuant des raids et incendiant des villages. Dans les villes, ils ne trouvèrent que des hommes âgés, des femmes et des enfants, qui, d'après un récit, «n'opposèrent aucune résistance; les hommes furent littéralement massacrés $»^{41}$. Moluntha, un chef âgé qui avait signé le traité, vint à la rencontre des Américains avec, dans la main, un exemplaire du traité et mourut avec, dans le crâne, une hache américaine ${ }^{42}$. Le traité de Hopewell avec les États-Unis en 1785 garantissait officiellement les limites cherokees mais Dragging Canoe n'y participa pas et de nombreux pionniers sur le front ne tinrent pas compte de ses termes; «Vos gens s'établissent bien plus vite sur nos terres après un traité qu'avant », comme le remarqua amèrement Old Tassel, le vieux

(39) Leonard J. SAdosky, Revolutionary Negotiations. Indians, Empires, and Diplomats in the Founding of America, Charlottesville, University Press of Virginia, 2010.

(40) Randolph C. Downes, Council Fires on the Upper Ohio, Pittsburgh, Pittsburgh University Press, 1940, p. 294 (citation). Les trois traités et documents relatifs sont reproduits dans Colin G. Calloway (dir.), Revolution and Confederation; Alden T. Vaughan (dir.), Early American Indian Documents Treaties and Laws, 1607-1789, 20 vol., Bethesda, MD, University Publications of America, 1979-2004, vol. 18, chap. 4.

(41) «Logan's Campaign - $1786 »$, Ohio Archaeological and Historical Publications, vol. 22, 1913, p. 520-521 ; The Military Journal of Major Ebenezer Denny, Philadelphia Lippincott, 1859 , p. 94.

(42) Colin G. Calloway, American Revolution in Indian Country..., op. cit., p. 175-177. 
chef cherokee ${ }^{43}$. Old Tassel travaillait pour la paix mais une milice américaine l'assassina sous un drapeau de trêve en 1788.

Des deux côtés, les dirigeants insistaient sur la modération mais les lignes de combat se durcissaient. Les pionniers sur la frontière ne tenaient pas compte des restrictions et dénonçaient les élites de l'Est qui semblaient placer les intérêts des Indiens au-dessus des leurs et ne parvenaient pas à protéger les colons contre les attaques des Indiens; les guerriers indiens s'élevaient contre des tactiques d'accommodements et créaient de nouveaux mouvements de résistance. Les commissaires américains qui rencontrèrent les Shawnees en conseil en 1792 remarquèrent que les guerriers étaient assis devant les chefs de villages plus âgés, ce qui illustrait le changement dans la manière traditionnelle de placer les participants selon laquelle les chefs civils avaient voix prépondérante. Les guerriers shawnees, âgés d'une vingtaine d'années ou au début de la trentaine à ce moment-là, n'avaient jamais connu la paix. Comme nombre de leurs voisins non indiens, ils vivaient dans une culture de violence ${ }^{44}$.

Face à l'afflux des colons et au développement d'une nation en marche, les Indiens reconstituèrent des coalitions multi-tribales et se battirent pour contenir l'expansion américaine à la rivière Ohio - essentiellement à la ligne de démarcation établie par le traité de Fort Stanwix pendant une douzaine d'années après la Révolution. Ce ne fut que lorsque le général Anthony Wayne vainquit les tribus alliées du Nord-Ouest à la bataille de Fallen Timbers en 1794 que les Indiens firent la paix. Au traité de Greenville en 1795, les chefs qui avaient combattu contre les Américains depuis l'époque de la Révolution cédèrent la plus grande partie de l'Ohio aux États-Unis. Mais la frontière du traité de Greenville ne fut pas plus efficace pour enrayer l'expansion américaine que ne l'avait été la proclamation ou les limites définies à Fort Stanwix. "Peu de choses, à part une muraille de Chine, ou une ligne de soldats, ne contiendra les intermédiaires immobiliers et les empiétements des colons sur le territoire indien », dit George Washington ${ }^{45}$. Une génération de guerriers plus jeunes se déplaça vers l'Ouest et poursuivit le combat, alors que les Indiens qui

(43) Cité par Colin G. Calloway, American Revolution in Indian Country..., op. cit., p. 209.

(44) Patrick Griffin, American Leviathan..., op. cit., David Andrew Nichols, Red Gentlemen and White Savages Indians, Federalists, and the Search for Order on the American Frontier, Charlottesville, University of Virginia Press, 2008; Colin G. CALlowaY, American Revolution in Indian Country..., op. cit., chap. 10.

(45) John C. FitzPATRick (dir.), The Writings of George Washington from the Original Manuscript Sources, 1745-1799, 39 vol., Washington, D.C., Government Printing Office, 1931-1944, vol. 25 , p. 112. 
restèrent dans leurs territoires survécurent en adoptant des formes plus subtiles de résistance culturelle et d'adaptation, conservant ce qu'ils pouvaient de leur vie et de leur culture dans une nation bien résolue à faire disparaître les deux.

George Washington, son secrétaire à la guerre Henry Knox, Thomas Jefferson et d'autres pères fondateurs débattirent de la question de savoir comment les Américains pouvaient traiter honorablement les Indiens au moment même où ils édifiaient leur nation sur des terres indiennes. Mais la prise de territoires indiens ne fut jamais discutée. Ayant gagné leur indépendance vis-à-vis de l'empire britannique à l'Est, les États-Unis se tournèrent vers l'Ouest pour construire ce que Jefferson nomma « un empire de liberté ». Dans cet empire, tous les citoyens partageaient les avantages. Mais - et ce fut une question qui, des générations durant, tourmenta la nation et la conscience nationale - qui avait qualité de citoyen?

Dans le cas des Indiens, la Déclaration d'Indépendance et la guerre de la Révolution sur la frontière donnèrent des réponses claires. Le document fondateur ainsi que les souvenirs de la guerre attribuèrent, tous, la cruauté et la violence des conflits dans l'arrière-pays aux Indiens, ces « sauvages » qui avaient démontré leur brutalité de nombreuses fois, et ne pouvaient faire partie de la vision d'égalité entre les hommes enchâssée dans la Déclaration. Ayant combattu contre les libertés et les droits américains au moment de la naissance de la nation, ils ne pouvaient s'attendre à partager ces droits et ces libertés qui avaient été gagnés par tant de sang versé. Alors que le gouvernement fédéral tentait de faire régner l'ordre à la place du chaos à l'Ouest, il mit en œuvre une politique nationale fondée sur le besoin de terres indiennes et sur l'hypothèse de la sauvagerie des Indiens. En tant que commandant de la milice du Tennessee, Andrew Jackson évoqua des souvenirs de guerriers indiens soutenus par les Britanniques, brandissant des couteaux à scalper et des tomahawks « contre nos femmes et enfants sans défense » au cours de la guerre révolutionnaire pour justifier son propre style de lutte sauvage contre les Indiens creeks; lorsqu'il fut président, il cita l'infériorité inhérente des Indiens et la fin inévitable de leur vie primitive pour justifier l'Indian Removal Act de $1830^{46}$. De même que Washington, Knox et Jefferson, des adminis-

(46) Jackson, cité dans Daniel K. RichTER, Facing East from Indian Country A Native History of Early America, Cambridge, Mass., Harvard University Press, 2001, 228; discours sur l'état de l'Union de Jackson, 6 décembre 1830, dans Theda Perdue et Michael D. GreEn (dir.), The Cherokee Removal. A Brief History with Documents, $2^{\mathrm{e}}$ éd., Boston, Bedford/St. Martin's, 2005, p. 127-128. 
trations ultérieures mirent en œuvre des programmes de " civilisation » dans leur politique indienne mais ils n'étaient pas très différents de ceux de Jackson pour l'essentiel; les Américains devaient avoir les terres indiennes et les « sauvages » devaient en être écartés, soit physiquement, soit culturellement.

Colin G. CallowaY Department of History and Native American Studies Program Dartmouth College Hanover, NH 03755

USA colin.calloway@dartmouth.edu 